\section{PRELIMINARY RESULTS OF A PHASE 2 STUDY OF INTRATUMORAL ADMINISTRATION OF BO-112 WITH PEMBROLIZUMAB IN PATIENTS WITH ADVANCED MELANOMA THAT HAVE PROGRESSIVE DISEASE ON ANTI-PD-1-BASED THERAPY}

${ }^{1}$ Iván Márquez Rodas*, ${ }^{2}$ Philippe Saiag, ${ }^{3}$ Luis de la Cruz Merino, ${ }^{4}$ Caroline Dutriaux, ${ }^{5}$ Juan Rodríguez-Moreno, ${ }^{6}$ Caroline Robert, ${ }^{7}$ Ana Arance, ${ }^{8}$ Eduardo Castañón Álvarez, ${ }^{9}$ Pablo Cerezuela-Fuentes, ${ }^{10}$ Henry Montaudie, ${ }^{8}$ Miguel Sanmamed, ${ }^{11}$ María González Cao, ${ }^{12} J$ ulie Charles, ${ }^{13}$ María Pilar López Criado, ${ }^{14}$ Alfonso Berrocal, ${ }^{1}$ Enrique de Miguel, ${ }^{2}$ Elisa Funk-Brentano, ${ }^{15}$ Sorilla Prey, ${ }^{7}$ Roberto Huertas, ${ }^{16}$ Delvys Rodríguez Abreu, ${ }^{17}$ Eva Muñoz Couselo, ${ }^{18}$ Juan Martin-Liberal, ${ }^{19}$ Javier Sánchez López, ${ }^{19}$ Helena Escuin-Ordinas, ${ }^{19}$ Marisol Quintero, ${ }^{19}$ Sonia Maciá, ${ }^{20}$ Marya Chaney, ${ }^{21}$ Stephane Dalle. ${ }^{1}$ Hospital Universitario Gregorio Marañón, madrid, Spain; ${ }^{2}$ Hôpital Ambrois Pare, Paris, France; ${ }^{3}$ Hospital Universitario Virgen Macarena, Sevilla, Spain; ${ }^{4}$ Hôpital Saint-André, Bourdeaux, France; ${ }^{5}$ Hospital Universitario Sanchinarro-Clara, Madrid, Spain; ${ }^{6}$ Hôpital Gustave Roussy, Villejuif-Paris, France; ${ }^{7}$ Hospital Clínic Barcelona, Barcelona, Spain; ${ }^{8}$ Clínica Universidad de Navarra, Madrid, Spain; ${ }^{9}$ Hospital Clínico Universitario Virgen de, Murcia, Spain; ${ }^{10}$ Centre Hospitalo-Universitaire de Nice, Nice, France; ${ }^{11}$ Hospital Universitari Dexeus, Barcelona, Spain; ${ }^{12}$ Centre Hospitalier Universitaire Grenobl, Grenoble, France; ${ }^{13} \mathrm{MD}$ Anderson, Madrid, Spain; ${ }^{14}$ Hospital General Universitario de Valenc, Valencia, Spain; ${ }^{15}$ Hôpital SaintAndré Bourdeaux, Bourdeaux, France; ${ }^{16}$ Hospital Universitario Insular de Gran C, Las Palmas de Gran Canaria, Spain; ${ }^{17}$ Hospital General Universitario Vall d'He, Barcelona, Spain; ${ }^{18}$ Catalan Institute of Oncology (ICO), Barcelona, Spain; ${ }^{19}$ Highlight Therapeutics, Valencia, Spain; ${ }^{20}$ Merck and Co., Inc, Kenilworth, NJ, USA; ${ }^{21}$ Hôpital Lyon Sud, Lyon, France

Background Intratumoral immunotherapies are being tested in different solid tumors. They trigger local and systemic responses. $^{1}{ }^{2}$ BO-112 is a double stranded RNA nanoplexed with polyethyleneimine (PEI), which mimics a viral infection and mobilizes the immune system.

In preclinical models and in a first in human clinical trial BO-112 activated dendritic cells, induced CD-8 infiltration, apoptosis and enhancement of immunogenic cell death and achieved an objective response in 2 out of 10 patients with melanoma with primary resistance to antiPD-1. ${ }^{3} 4$

Methods In this phase 2 study, BO-112 plus pembrolizumab is evaluated in patients with advanced melanoma, who have developed progressive disease while on or within 12 weeks after anti-PD1/PD-L1 based therapy (either as first line or as adjuvant treatment). BO-112 is administered intratumorally once weekly in 1 to 8 tumor lesions, total dose 1 to $2 \mathrm{mg}$, for the first 7 weeks and thereafter every three weeks; pembrolizumab $200 \mathrm{mg}$ is administered intravenously every three weeks. Overall response rate (ORR) is analyzed as primary endpoint by independent reviewer. Secondary objectives include disease control rate (DCR), duration of response and progression free survival (PFS); response assessment is done by RECIST 1.1 and iRECIST; in addition, CD-8 and PD-L1 IHC, NGS, itRECIST and radiomics signatures are prospectively assessed. Key eligibility criteria include cutaneous or mucosal melanoma with known BRAF status; at least one lesion RECIST 1.1 measurable and amenable for IT injection. Enrollment has been completed on 26th August.

Results With 26 evaluable patients with a first response assessment, seven have progressive disease (PD), five have partial response (PR) and fourteen patients show stable disease (SD). Preliminary ORR is $19.2 \%$ and DCR is $73.1 \%$ at week 8 . Three patients with PR at week 8 have undergone a second assessment at week 16, with further decrease in sum of diameters (SOD) in both injected and non-injected lesions. Three out of five patients with SD and a second assessment maintain $\mathrm{SD}$, showing a decrease in SOD in two cases (figure 1). In addition, two patients with only skin lesions have a pathological complete response. CD8 and PD-L1 have increased in 8 and 7 out of 13 patients with paired biopsies, being related with clinical benefit (figure 2).

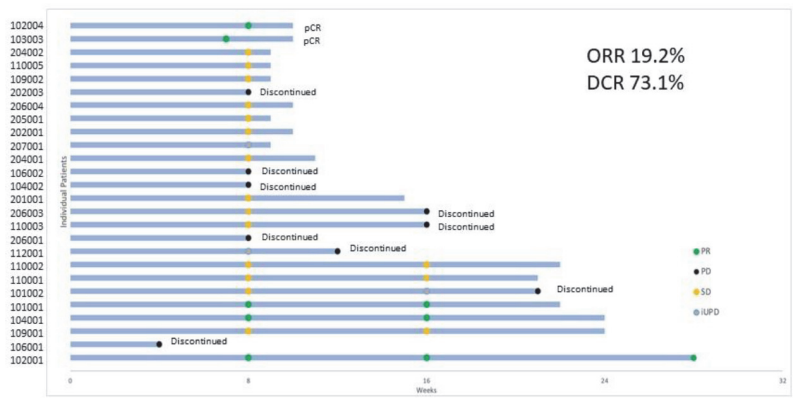

Abstract 961 Figure 1 Swimmer plot, efficacy data for evaluable patients undergoing at least one response assessment
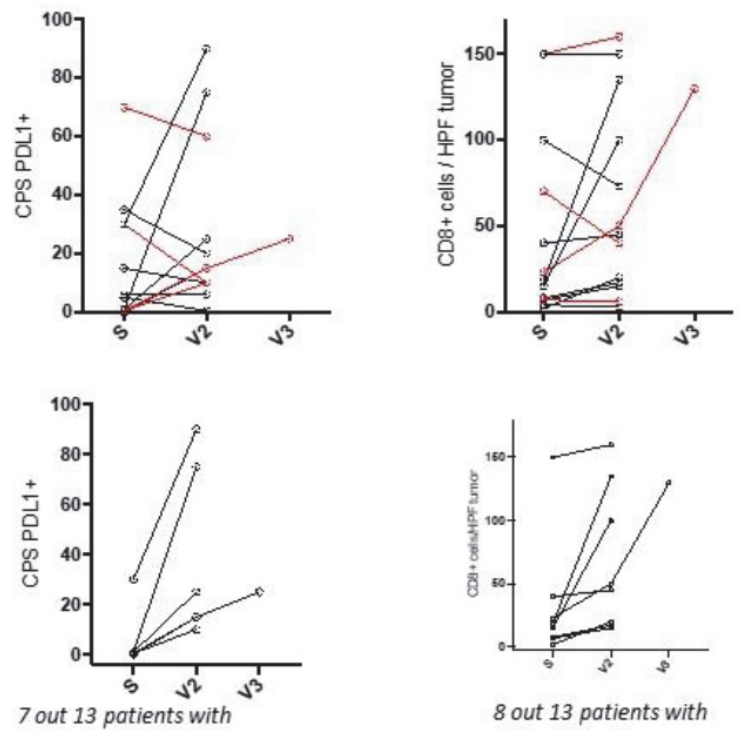

CPS PDI 1+ expression up
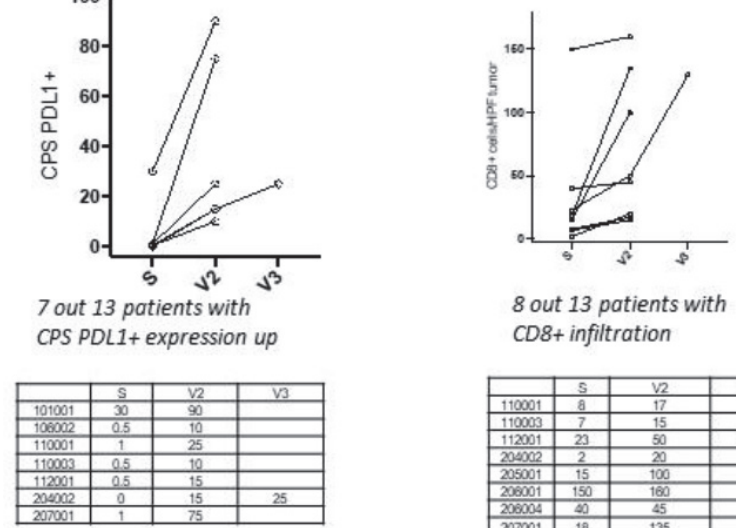

8 out 13 patients with CD8+ infiltration

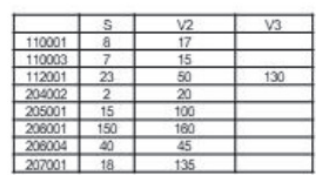

Abstract 961 Figure 2 Immunohistochemistry data for CPS and CD8 data from paired biopsies

Conclusions Despite these data being preliminary, there is a trend for benefit in terms of ORR and also in long lasting stable diseases. $\mathrm{BO}-112$ is able to increase PD-L1 expression in tumor cells and increase CD8-T cell infiltrates.

Acknowledgements Merck, Pivotal SLU, Quibim radiomics, Pangaea laboratories, all participating sites and patients

Trial Registration NCT04570332

\section{REFERENCES}

1. Aznar MA, Planelles $L$, Perez-Olivares $M$, et al. Immunotherapeutic effects of intratumoral nanoplexed poly I:C. J Immunother Cancer. 2019 May 2;7(1):116.

2. 5. Hamid 0, Ismail R, Puzanov I. Intratumoral immunotherapy-update 2019. The Oncologist 2020;25:e423-438.

3. Márquez-Rodas I, Longo F, Rodríguez-Ruiz $\mathrm{M}$, et al. Intratumoral nanoplexed poly I:C BO-112 in combination with systemic anti-PD-1 for patients with anti-PD-1refractory tumors. Sci Transl Med 2020 Oct 14;12(565):eabb0391. 


\section{Abstracts}

4. Kalbasi A, Tariveranmoshabad M, Hakimi K, Kremer S, et al. A. Uncoupling interferon signaling and antigen presentation to overcome immunotherapy resistance due to JAK1 loss in melanoma. Sci Transl Med 2020 Oct 14;12(565):eabb0152.

Ethics Approval The study obtained ethics approval by Spanish Health Agency (AEMPS), on 11th December 2020, and French Health Agency (ANSM) on 27th January 2021; study obtained approval from two Ethics Committee: Vall D'Hebron, Barcelona, Spain on 7th December 2020 (number 467), and Centre Léon Bérard, Lyon, France, CPP 20.11.10.38825 on 11th February 2021.

For each study patient, written informed consent is obtained prior to any protocol-related activities. As part of this procedure, the principal investigator or one of his/her associates must explain orally and in writing the nature, duration, and purpose of the study, and the action of the study drug in such a manner that the patient is aware of the potential risks, inconveniences, or adverse effects that may occur. They should be informed that the patient may withdraw from the study at any time. They will receive all information that is required by the regulatory authorities and $\mathrm{ICH}$ guidelines. The ICF has been signed by the patient and a copy provided to them.

\section{Consent N/A}

http://dx.doi.org/10.1136/jitc-2021-SITC2021.961 